INPLASY

PROTOCOL

To cite: Rosa et al.

Levonorgestrel-releasing

intrauterine system use and

Risk of Breast cancer:

systematic Review and metaanalysis. Inplasy protocol

202030002. doi:

10.37766/inplasy2020.3.0002

Received: 13 March 2020

Published: 15 March 2020

Corresponding author:

Maria Ines da Rosa

mir@unesc.net

Author Affiliation: University of the extreme south

Catarinense

Support: No

Review Stage at time of this submission: Preliminary searches.

Conflicts of interest: No

\section{Levonorgestrel-releasing intrauterine system use and Risk of Breast cancer: systematic Review and meta-analysis}

Rosa Ml1; Silva, F2; Grande, A3; Macedo, A4; Colonetti T5; Costa, $\mathrm{M}^{6}$.

\section{ABSTRACT}

Review question: To assess the risk of levonorgestrelreleasing intrauterine system use to breast cancer and recurrence of breast cancer P: Pre- and postmenopausal women : I: (1) women without breast cancer using LNG-IUS in observational studies and risk of breast cancer (2) women with breast cancer on adjuvant tamoxifen using LNG-IUS, in RCTs studies and risk of breast cancer recurrence. C: Control Group O: risk of breast cancer recurrence

Methods: The searches were conducted from inception up to September 2019 in: Cochrane Library; Meline, EMBASE, ; LILACS; and Google Scholar. We also searched references of relevant studies, using the following terms: (("levonorgestrel"[MeSH Terms] OR "levonorgestrel"[All Fields]) AND intrauterine[All Fields] AND system[All Fields] AND LNG[All Fields] AND IUS[All Fields] AND ("breast neoplasms"[MeSH Terms] OR ("breast"[All Fields] AND "neoplasms"[All Fields]) OR "breast neoplasms"[All Fields] OR ("breast"[All Fields] AND "cancer"[All Fields]) OR "breast cancer"[All Fields]) OR "recurrence breast cancer" [All Fields]) AND ("tamoxifen"[MeSH Terms] OR "tamoxifen"[All Fields])) to search relevant studies. The research was limited to humans, regardless of the language of the studies analyzed. The lists of references of all the selected studies were checked as well as the gray literature.

INPLASY registration number: This protocol was registered with the International Platform of Registered Systematic Review and Meta-Analysis Protocols (INPLASY) on 15 March 2020 and was last updated on 15 March 2020 (registration number INPLASY202030002.

\section{INTRODUCTION}

Objectives / Review question: To assess the risk of levonorgestrel-releasing intrauterine system use to breast cancer and recurrence of breast cancer P: Pre- and postmenopausal women : I: (1) women without breast cancer using LNG-IUS in observational studies and risk of breast cancer (2) women with breast cancer on adjuvant tamoxifen using LNG-IUS, in RCTs studies and risk of breast cancer 
recurrence. C: Control Group O: risk of breast cancer recurrence.

Condition being studied: Breast cancer is the most commonly occurring cancer in women and the second most common cancer overall. There were over 2 million new cases in 2018 (Bray et al 2018). The initiation of discussion of the potential role of progesterone associated with breast cancer risk occurred after the publication of Women's Health Initiative study (2002), in which a significant association was shown in crude HR = 1.26 (Nominal $95 \% \mathrm{Cl}$, 1.00-1.59) but which in the adjusted analysis did not show statistical significance (Adjusted 95\% Cl, 0.83-1.92). (Rossouw et al 2002). Progesterone in the breast is proliferative and the action is in conjunction with estrogen and estrogen receptors (ER) in the expansion of glandular structures during development (Brisken and O'Malley 2010). The levonorgestrel-releasing intrauterine system began use in 1990 in Europe and in 2000, in the United States. Levonorgestrel is a second generation progestogen that thickens cervical mucus and suppresses endometrial proliferation creating a hostile environment for sperm survival by inhibiting motility. It also promotes a thinning of the endometrium preventing implantation of the embryo in case of fertilization. Due to this action, it is commonly used as endometrial cancer prophylaxis in women with breast cancer using tamoxifen(ESHRE, 2008).

\section{METHODS}

Participant or population: 1) Pre- and postmenopausal women without breast cancer using LNG-IUS 2) Pre- and postmenopausal women with breast cancer on adjuvant tamoxifen using LNGIUS.

Intervention: women without breast cancer using LNG-IUS 2) women with breast cancer on adjuvant tamoxifen using LNGIUS.

\section{Comparator: Control Group}

Study designs to be included: We included randomized controlled trials and observational studies : cohort prospective or retrospective, case-control and crosssectional studies.

Eligibility criteria: women without breast cancer using LNG-IUS 2) women with breast cancer on adjuvant tamoxifen using LNG-IUS.

Information sources: The searches were conducted from inception up to September 2019 in: Cochrane Library; Meline, EMBASE, ; LILACS; and Google Scholar. We also searched references of relevant studies, using the following terms: (("levonorgestrel"[MeSH Terms] OR "levonorgestrel"[AII Fields]) AND intrauterine[All Fields] AND system[AII Fields] AND LNG[All Fields] AND IUS[AII Fields] AND ("breast neoplasms"[MeSH Terms] OR ("breast"[All Fields] AND "neoplasms"[All Fields]) OR "breast neoplasms"[All Fields] OR ("breast"[All Fields] AND "cancer"[All Fields]) OR "breast cancer"[All Fields]) OR "recurrence breast cancer" [All Fields]) AND ("tamoxifen" [MeSH Terms] OR "tamoxifen"[All Fields])) to search relevant studies. The research was limited to humans, regardless of the language of the studies analyzed. The lists of references of all the selected studies were checked as well as the gray literature.

Main outcome(s): Assess the risk of levonorgestrel-releasing intrauterine system use to breast cancer.

Quality assessment / Risk of bias analysis: All included studies will be assessed for their methodological quality. The Cochrane Collaboration's risk of bias tool will be used (RevMan 5.3) for RCT studies. The criteria consist of 7 items: random sequence generation, allocation concealment, blinding of participants and researchers, blinding of outcome assessment, incomplete outcome data, selective outcome reporting, and other sources of bias. For observational studies the Newcastle-Ottawa Scale will be used. 
Strategy of data synthesis: For both outcomes analyzed, breast cancer and Breast cancer recurrence, we expressed the results as OR with $95 \%$ confidence intervals $(95 \% \mathrm{Cl})$ and combined for metaanalysis with ReviewManager5 software using a randomic-effect model. (Higgins 2011). In order to investigate heterogeneity, the $\mathrm{X}^{2}$ test was used and for inconsistency the I 2 test. The $\left.\right|^{2}$ statistic, which represents the overall heterogeneity that includes clinical, method, and statistical components, was used to select the appropriate effect model for pooling estimates. $I^{2}$ value between $0 \%$ and $25 \%$ indicates no heterogeneity, $26 \%$ and $50 \%$ indicates low heterogeneity, $51 \%$ and $75 \%$ indicates moderate heterogeneity, and $76 \%$ and $100 \%$ indicate high heterogeneity. The quality of evidence produced was evaluated using the Grading of Recommendations Assessment, a summary of findings table using GRADEpro soft-ware.

Subgroup analysis: No

Sensibility analysis: If necessary, an analysis of the sensitivity.

Countries involved: Brazil

Keywords: levonorgestrel; brest cancer; tamoxifen.

Contributions of each author:

1 - Not reported

2 - Project, study selection, data extraction and article writing.

3 - Study selection, data extraction, data analysis and writing.

4 - Study selection, data extraction, data analysis and writing.

5 - Study selection, data extraction, data analysis and writing.

6 - study selection and writing. 